\title{
Pilomatrixkarzinom mit lymphogener Metastasierung - ein Fallbericht
}

\author{
T. Stern ${ }^{1}$ \\ H. Röckmann ${ }^{1,2}$ \\ E. Dippel ${ }^{1}$ \\ W. Back $^{3}$ \\ D. Schadendorf ${ }^{1,2}$ \\ S. Goerdt ${ }^{1}$
}

\section{Pilomatrix Carcinoma with Lymphogenic Metastases - A Case Report}

\section{Zusammenfassung}

Das Pilomatrixkarzinom als maligne Variante des Pilomatrixoms ist ein sehr seltener epithelialer Tumor mit Haarmatrixdifferenzierung. Seit seiner Erstbeschreibung wurden zirka 80 Fälle, in etwa $10 \%$ davon mit Metastasierung, publiziert. Wir berichten über einen 83-jährigen Mann, der vor 3 Jahren erstmals einen Tumor am Rücken bemerkt hatte. Nach mehrfachen auswärtigen Exzisionen war es jeweils zu Lokalrezidiven gekommen. Die histologischen Untersuchungen ergaben unterschiedliche Resultate (M. Bowen, Plattenepithelkarzinom, Merkelzellkarzinom). Bei der Erstvorstellung in unserer Klinik fielen bereits vergrößerte axilläre Lymphknoten auf. Nach Exzision des erneuten lokalen kutanen Tumorrezidivs und einer Lymphknotenmetastase konnte schließlich die Diagnose eines lymphogen metastasierten Pilomatrixkarzinoms gesichert werden. Aufgrund dieser differenzialdiagnostischen Schwierigkeiten werden hier die relevanten klinischen und histomorphologischen Kriterien zur Unterscheidung des Pilomatrixkarzinoms vom Pilomatrixom, aber auch zur Abgrenzung von anderen epithelialen Tumoren diskutiert. Aufgrund der geringen Inzidenz des Pilomatrixkarzinoms gibt es keinen etablierten Therapiestandard, es wird jedoch die radikale Exzision des Tumors mit einem Sicherheitsabstand von mindestens $5 \mathrm{~mm}$ empfohlen.

\section{Abstract}

Pilomatrix carcinoma, the malignant counterpart of pilomatrixoma, is a very rare epithelial tumor with hair matrix differentiation. Since its first description about 80 cases, of which approximately 10 percent were metastatic, have been published. We report here a case of an 83 year old man, who for the first time noticed a tumor on his back in 2001. After several surgical excisions local recurrences appeared in short intervals. The histological examinations resulted in widely different diagnoses. At his first visit to our hospital his axillary lymph nodes were already palpable. After excision of the newly relapsed skin tumor and of the lymph node metastasis the diagnosis of a pilomatrix carcinoma was confirmed. Due to these differentiating difficulties the relevant clinical and histomorphological criteria distinguishing pilomatrix carcinoma and pilomatrixoma, as well as their distinction from other epithelial tumors, are discussed. Because of the small incidence of the pilomatrix carcinoma there is no established therapy standard, however the radical surgical excision of this neoplasm with a safety margin of at least $5 \mathrm{~mm}$ is recommended.

Institutsangaben

${ }^{1}$ Klinik für Dermatologie, Venerologie und Allergologie (Direktor: Prof. Dr. S. Goerdt)

${ }^{2}$ Klinische Kooperationseinheit für Dermato-Onkologie des DKFZ (Leiter: Prof. Dr. D. Schadendorf), an der Klinik für Dermatologie, Venerologie und Allergologie

${ }^{3}$ Pathologisches Institut (Direktor: Prof. Dr. U. Bleyl), Klinikum Mannheim gGmbH, Universitätsklinikum, Ruprecht-Karls-Universität Heidelberg, Mannheim

Korrespondenzadresse

T. Stern · Klinik für Dermatologie, Venerologie und Allergologie · Klinikum Mannheim gGmbH ·

Universitätsklinikum · Theodor-Kutzer-Ufer 1 - 3 · 68167 Mannheim ·

E-mail: thorsten.stern@haut.ma.uni-heidelberg.de

Bibliografie

Akt Dermatol 2004; 30: 322-326 @ Georg Thieme Verlag Stuttgart KG · New York

DOI 10.1055/s-2004-825681 · ISSN 0340-2541 


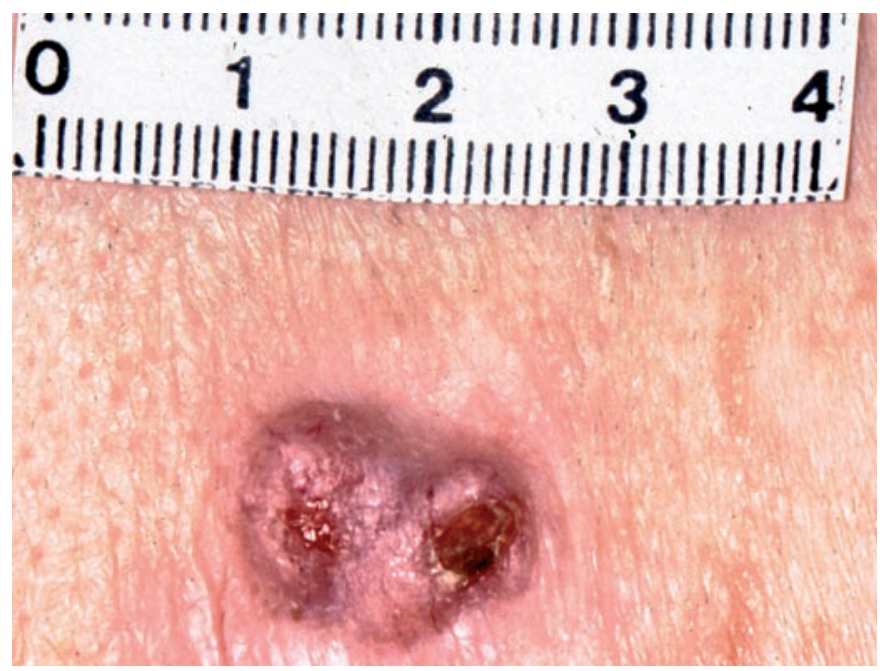

Das Pilomatrixkarzinom ist ein sehr seltener maligner epithelialer Tumor mit Haarmatrixdifferenzierung, der sich klinisch durch sein lokal aggressives Wachstumsmuster und seine Metastasierungstendenz, bei genauer Analyse auch histologisch, von seiner benignen Variante, dem Pilomatrixom, unterscheidet [1]. Das Pilomatrixom wurde erstmals 1880 von Malherbe und Chenantais als „epitheliome calcifié des glandes sebacées“ beschrieben [2]. Später konnte nachgewiesen werden, dass es sich bei dieser Entität um einen Tumor des Epithels der Haarzellmatrix handelt, so dass von Forbis und Helwig 1961 die noch heute gebräuchliche Bezeichnung „Pilomatrixom“ eingeführt wurde [3,4]. Bereits 1927 wurde von Gromiko erstmals eine maligne Variante dieses Tumors beschrieben, für die schließlich 1980 von Lopransri und Mihm der Terminus „Pilomatrixkarzinom“ geprägt wurde $[5,6]$. Seit der Erstbeschreibung eines Pilomatrixkarzinoms im Jahre 1927 wurden erst zirka 80 Fälle dieses malignen Tumors publiziert. In etwa $10 \%$ konnte eine Metastasierung dokumentiert werden [7-41]. Wir berichten über ein Pilomatrixkarzinom, das bei definitiver Diagnosestellung bereits in die regionären axillären Lymphknoten metastasiert hatte.

\section{Kasuistik}

\section{Anamnese}

Der hier vorgestellte 83-jährige Patient hatte erstmals vor zirka 3 Jahren einen Hauttumor am Rücken bemerkt. Nach zweimaliger Exzision mit unbekanntem Sicherheitsabstand war es jeweils zu einem Lokalrezidiv gekommen. Die histologischen Untersuchungen der Exzisate ergaben mit Morbus Bowen bzw. Plattenepithelkarzinom jeweils unterschiedliche Ergebnisse. Nach einem erneuten Lokalrezidiv wurde im Juli 2003 nochmals eine Probeexzision mit der histologischen Diagnose eines Merkelzellkarzinoms durchgeführt. Im August 2003 wurde der Patient dann erstmals in der Klinik für Dermatologie, Venerologie und Allergologie des Universitätsklinikum Mannheim vorgestellt.

\section{Dermatologischer und körperlicher Aufnahmebefund}

Es zeigte sich paravertebral rechts in Höhe des Dermatoms Th 12 ein $2,5 \times 2 \mathrm{~cm}$ großer erythematöser Nodus mit krustig belegten Ulzerationen. Der Tumor war nicht druckschmerzhaft und schlecht verschieblich (Abb.1).

Bei der sonst unauffälligen körperlichen Untersuchung waren axillär rechts vergrößerte Lymphknoten palpabel.

\section{Apparative Untersuchungen}

Lymphknotensonographie: axillär rechts $4,3 \times 2,5 \mathrm{~cm}$ große echogene Raumforderung, sonst unauffällig. Beurteilung: dringender Verdacht auf eine Lymphknotenmetastase.

Sonographie des Abdomens: sonomorphologisch unauffälliger Befund.

CT des Thorax mit Kontrastmittel: teils nekrotisierend zerfallenes oder partiell eingeblutetes Lymphom axillär rechts mit einer maximalen Ausdehnung von 4,6 ×3,1 ×5,5 cm; sonst kein Anhalt für weitere Filiae.
Abb. 1 Lokalbefund am Rücken.

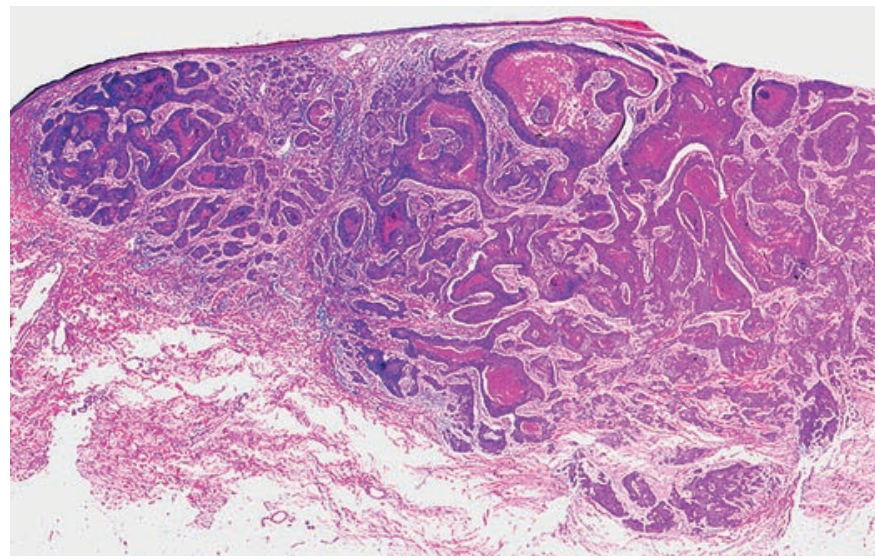

Abb. 2 Histologischer Schnitt Hautexzisat.

MRT des Gehirns mit Kontrastmittel: keine Filiae nachweisbar.

\section{Histologische Befunde}

Abb. 2 zeigt einen histologischen Schnitt des Hautexzisats mit einem knotigen, breitbasigen Tumor im Bereich des Rückens. Die Tumormassen reichten verzweigt und knotenartig bis in die tiefe Subkutis. Der Tumor war aufgebaut aus mittelgroßen basaloiden Zellen mit zum Teil erheblicher Zell- und Kernpleomorphie und vermehrten Mitosefiguren. Weiterhin zeigten sich zentrale ausgedehnte Nekrosezonen. In der Vergrößerung (Abb.3) erkennt man die für das Pilomatrixkarzinom charakteristischen basaloiden Tumorzellkomplexe im Randbereich und die so genannten Schattenzellen mit graduellem Verlust des Zellkerns im Zentrum. In einer weiteren immunhistochemischen Untersuchung zeigten die Tumorzellen eine negative Reaktion auf Antikörper gegen Zytokeratin 20 und Chromogranin A und eine positive Reaktion auf Antikörper gegen Zytokeratin 5/6, so dass auch der nostizierten Merkelzellkarzinoms entsprach.

Im Exzisat der rechten Axilla fand sich neben fünf tumorfreien Lymphknoten eine bis zu $7 \mathrm{~cm}$ große Metastase des Pilomatrixkarzinoms. Auch hier zeigten sich zahlreiche, unregelmäßig konimmunhistochemische Befund nicht dem Bild des zuvor diag- 


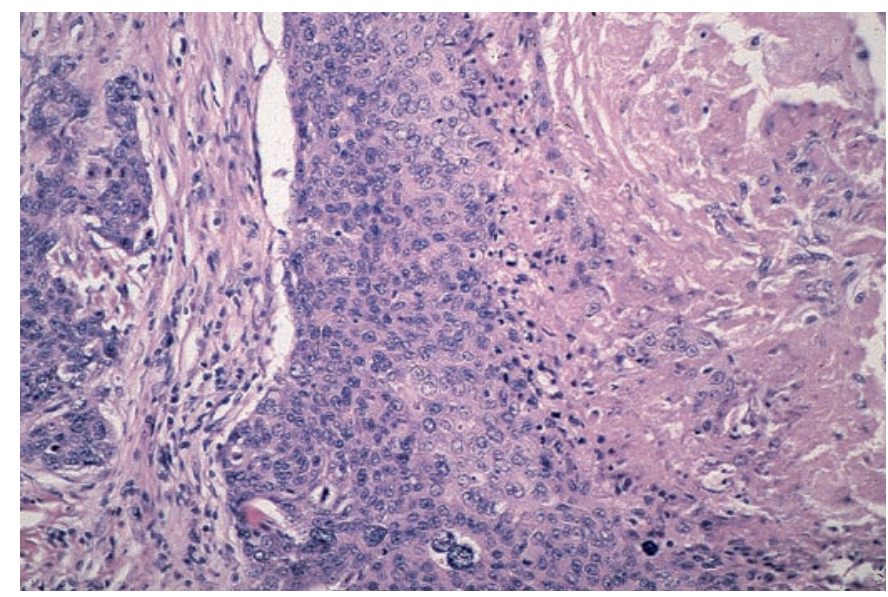

Abb. 3 Histologischer Schnitt Hautexzisat.

figurierte basaloide Tumorzellkomplexe, sowie Areale mit ausgedehnten Zellatypien. Daneben fanden sich zentral Schattenzellen und ausgedehnte Nekroseareale. Die typische Lymphknotenarchitektur war durch die massive Infiltration des Tumors gänzlich aufgehoben (Abb.4).

\section{Therapie und Verlauf}

Wir führten in Tumeszenzanästhesie eine radikale Exzision des Hauttumors mit einem Sicherheitsabstand von $1 \mathrm{~cm}$ und in gleicher Sitzung den Verschluss des Defektes mittels Rotationsplastik durch. Der postoperative Verlauf gestaltete sich komplikationsfrei. Aufgrund des dringenden Verdachts auf eine Metastasierung in die regionären Lymphknoten axillär rechts wurde anschließend eine Axilladissektion durch die Chirurgische Klinik mit o. g. histologischen Befunden komplikationslos durchgeführt. Nach Abheilung der Wundverhältnisse erhielt der Patient zur Rezidivprophylaxe eine fraktionierte Radiatio der rechten Axilla mit einer Gesamtdosis von $50 \mathrm{~Gy}$. Im weiteren Verlauf über jetzt 9 Monate war der Patient bis auf ein Serom in der rechten Achsel und ein Lymphödem des rechten Armes beschwerdefrei, insbesondere ergab sich klinisch kein Anhalt für ein Rezidiv oder für eine weitere Metastasierung des Pilomatrixkarzinoms. Eine im Dezember 2003 durchgeführte CT-Kontrolle war ebenfalls unauffällig.

\section{Diskussion}

Das Pilomatrixkarzinom stellt die maligne Variante eines Pilomatrixoms dar. Es kann primär oder auch auf dem Boden eines Pilomatrixoms entstehen [1]. Bislang wurden in der Literatur etwa 80 Fälle eines Pilomatrixkarzinoms publiziert [7-41]. Sau et al. stellten in ihrer Arbeit bereits publizierten Pilomatrixkarzinomen 19 eigene, zumeist retrospektiv diagnostizierte Fälle gegenüber [25,41]. Dieser große Anteil von erst retrospektiv erkannten Pilomatrixkarzinomen deutet auf die sowohl klinisch als auch histopathologisch schwierige Diagnosefindung hin. Der Tumor wird häufig erst im Laufe der Zeit nach Lokalrezidiven oder bei Metastasierung als maligne eingestuft und retrospektiv korrekt histologisch diagnostiziert [41]. Häufig werden Pilomatrixkarzinome klinisch als Pilomatrixome, Epidermalzysten oder als Basalzellkarzinome verkannt [39]. Auch in unserem Fall ist

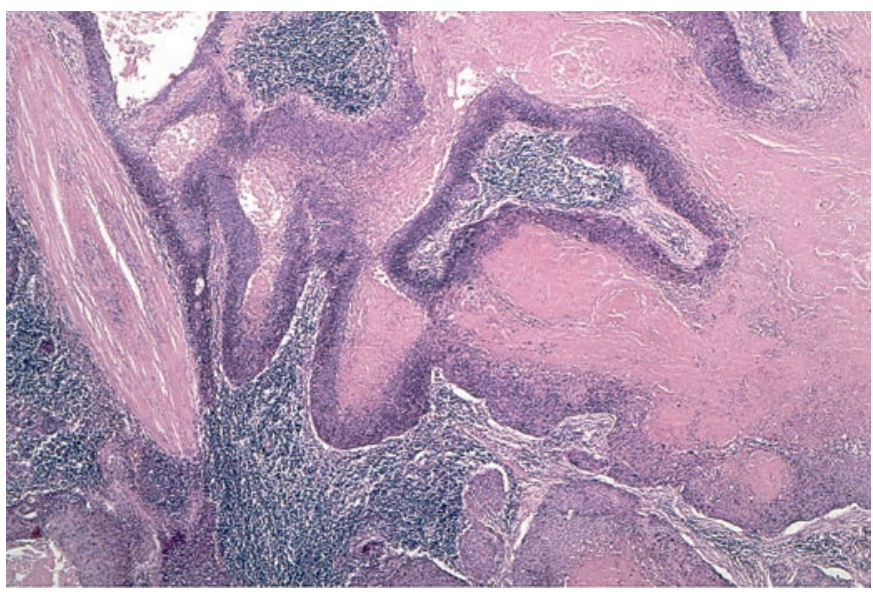

Abb. 4 Histologischer Schnitt axillärer Lymphknoten.

davon auszugehen, dass es sich bei dem Tumor des Patienten primär um ein Pilomatrixkarzinom gehandelt hat. In Tab.1 sind klinische Kriterien zusammengefasst, die für die Differenzialdiagnose zwischen Pilomatrixom und Pilomatrixkarzinom hilfreich sein können [41]. Das Pilomatrixkarzinom ist eine Neoplasie, die eher Männer im höheren Lebensalter betrifft, während das Pilomatrixom innerhalb der ersten beiden Lebensjahrzehnte auftritt und eine leichte Gynäkotropie aufweist. Auffällig ist weiterhin eine fämiliäre Häufung des Pilomatrixoms, insbesondere beim Gardner-Syndrom oder der myotonischen Dystrophie [6]. Ein deutliches Zeichen stellt das Lokalrezidiv dar. Sicherstes Kriterium der Malignität ist die Metastasierung, deren Häufigkeit in der Literatur mit 10 bis $20 \%$ angegeben wird [7,39]. In Tab. 2 findet sich eine Literaturübersicht der publizierten metastasierten Pilomatrixkarzinome. Erwähnenswert ist die in 6 von 11 Fällen (55\%) dokumentierte Streuung in die Lunge, an 2. Stelle folgt die lymphogene Metastasierung [7].

Neben der schwierigen klinischen Differenzialdiagnose zwischen Pilomatrixom und Pilomatrixkarzinom bereitet auch die histologische Unterscheidung große Schwierigkeiten. Auch im vorgestellten Fall wurden wechselnde histologische Diagnosen gestellt. Sowohl das Pilomatrixom als auch das Pilomatrixkarzinom zeigen Zeichen der Haarmatrixdifferenzierung. Beide weisen Gebiete mit so genannten Schattenzellen auf. Das sind keratinisierte eosinophile Zellen mit schwach angefärbten Kernen. Weiterhin sind basaloide Tumorzellkomplexe typisch für beide Tumoren. Das Pilomatrixkarzinom hingegen weist zusätzlich mindestens eines der folgenden Kriterien auf: eine hohe Mitoserate mit atypischen Mitosen, große pleomorphe hyperchromatische Zellkerne und das Auftreten einer vaskulären oder lymphogenen Infiltration [1,6] (Tab. 3). Histologisch und immunhistochemisch abzugrenzen sowohl vom Pilomatrixom als auch vom Pilomatrixkarzinom sind andere Tumoren der Haarfollikel, wie z.B. das Trichoepitheliom oder Basalzellkarzinome mit Matrixdifferenzierung [7]. Dagegen sind immunhistochemische Untersuchungen bei der Abgrenzung eines Pilomatrixkarzinoms von einem Pilomatrixom nicht hilfreich, da beide Läsionen ein ähnliches Anfärbeverhalten mit Zytokeratinen, EMA, CEA, S-100 und $\beta 2-M i k r o g l o b u l i n e n$ aufweisen [18]. Eine Typisierung des Pilomatrixoms bzw. des Pilomatrixkarzinoms bzgl. der Expression der neuen Haarkeratine steht allerdings noch aus. 
Tab 1 Klinische Kriterien zur Differenzialdiagnose; modfiziert nach Mack et al. [41]

\begin{tabular}{|lll}
\hline & Pilomatrixom & Pilomatrix-CA \\
\hline Alter & $<20$ Jahre & $>40$ Jahre \\
\hline Ges.verteilung $(\mathrm{m}: \mathrm{w})$ & $2: 3$ & $4: 1$ \\
\hline Lokalisation & Kopf, Hals, Arme & Kopf, Nacken, Rücken \\
\hline multiple TU & beschrieben & nicht beschrieben \\
\hline fam. Häufung & beschrieben & nicht beschrieben \\
\hline Rezidive & selten & häufig $(50-60 \%)$ \\
\hline Metastasierung & keine & möglich $(10-20 \%)$ \\
\hline
\end{tabular}

Tab. 2 Literaturübersicht metastasierter Pilomatrixkarzinome; modifiziert nach De Galvez et al. [7]

\begin{tabular}{|lll|}
\hline Jahr & Autor & Metastasen \\
\hline 1984 & Gould et al. & Lunge \\
\hline 1986 & Mir et al. & Lunge, Axilla-LK \\
\hline 1992 & Tateyama et al. & inguinale LK \\
\hline 1993 & O’Donovan et al. & Knochen \\
\hline 1994 & Hanly et al. & Lunge \\
\hline 1994 & Mack et al. & inguinale LK, Lunge, Knochen \\
\hline 1995 & Monchy et al. & submand. LK \\
\hline 1996 & Niedermayer et al. & Lunge, Hirn \\
\hline 1997 & Li et al. & Leber \\
\hline 2001 & De Galvez et al. & Lunge, ing. LK \\
\hline 2004 & Stern et al. & axillärer LK \\
\hline
\end{tabular}

Tab. 3 Histomorphologische Kriterien zur Differentialdiagnose; modifiziert nach Mack et al. [41]

\begin{tabular}{|c|c|c|}
\hline & Pilomatrixom & Pilomatrix-CA \\
\hline TU-Randzone & zirkumskript & $\begin{array}{l}\text { unregelmäßig, lokal } \\
\text { infiltrativ }\end{array}$ \\
\hline $\begin{array}{l}\text { basaloide } \\
\text { Zellgruppen }\end{array}$ & vor allem peripher & unregelmäßig verteilt \\
\hline TU-Zytologie & $\begin{array}{l}\text { uniform; kl. Zellkern, } \\
\text { unauffälliger Nukleolus }\end{array}$ & $\begin{array}{l}\text { pleomorph; vesikulärer } \\
\text { Zellkern mit gr. Nukleolus }\end{array}$ \\
\hline $\begin{array}{l}\text { klarzellige } \\
\text { Veränderungen }\end{array}$ & selten & häufig \\
\hline Nekrosen & selten & häufig \\
\hline atypische Mitosen & keine & möglich \\
\hline Verhornung & geordnet, zentral & unregelmäßig \\
\hline $\begin{array}{l}\text { infiltratives } \\
\text { Wachstum }\end{array}$ & $\begin{array}{l}\text { keine perineurale oder } \\
\text { vaskuläre Infiltrate }\end{array}$ & $\begin{array}{l}\text { perineurale und } \\
\text { vaskuläre Infiltrate }\end{array}$ \\
\hline
\end{tabular}

Aufgrund der geringen Inzidenz des Pilomatrixkarzinoms gibt es keinen etablierten Therapiestandard $[39,40]$. Ausgehend von den Daten aus der Literatur wird eine Exzision mit einem hinreichend großen, allerdings nicht genau definierten, Sicherheitsabstand empfohlen, da dieses Vorgehen mit einer geringeren Lokalrezidivrate assoziiert sein soll. Bei einer „weiten“ Exzision kam es dennoch in $50 \%$ der Fälle zu einem Lokalrezidiv, während bei einer „einfachen“ Exzision die Rezidivrate auf bis zu 67\% anstieg [38]. In einer weiteren Arbeit wurde bei einem Sicherheitsabstand von 5 bis $10 \mathrm{~mm}$ kein Lokalrezidiv beobachtet, so dass in Abhängigkeit von der Lokalisation geraten wird, einen Sicherheitsabstand von mindestens $5 \mathrm{~mm}$ zu wählen [39]. Kann dieser Sicherheitsabstand aufgrund z.B. anatomischer Gegebenheiten nicht eingehalten werden, ist auch eine Radiotherapie in Erwägung zu ziehen. So konnte bei 4 Patienten, die entweder im Vorfeld oder postoperativ bestrahlt wurden, kein Lokalrezidiv festgestellt werden $[16,25]$.

Für das Stadium der (Fern-)Metastasierung liegen keine Erfahrungswerte vor. Ein Patient, bei dem allerdings erst post mortem im Rahmen der Autopsie die Diagnose eines Pilomatrixkarzinoms gestellt werden konnte, erhielt eine kombinierte Interferon-Vinblastin-Chemotherapie, ohne dass das Tumorleiden aufgehalten werden konnte [41].

Wir möchten mit der Vorstellung dieses Falles auf die häufig schwierige klinische und histopathologische Diagnosestellung des Pilomatrixkarzinoms aufmerksam machen und den Blick auf diese seltene Neoplasie der Haut lenken.

\section{Literatur}

${ }^{1}$ Dutta R, Boadle R, Ng T. Pilomatrix carcinoma: case report and review of literature. Pathology 2001; 33: 248-251

${ }^{2}$ Malherbe A, Chenantais J. Note sur l'épitheliome calcifié des glandes sebacées. Bull Soc Anat Paris 1880; 5: 169-175

${ }^{3}$ Lever W, Griesemer R. Calcifying epithelioma of Malherbe. Arch Dermatol 1949; 59: 506-518

${ }^{4}$ Forbis R, Helwig EB. Pilomatrixoma (Calcifying Epithelioma). Arch Dermatol 1961; 83: 606-618

${ }^{5}$ Gromiko N. Zur Kenntnis der bösartigen Umwandlung des verkalkten Hautepithelioms. Virchows Archiv 1927; 205: 103 - 116

${ }^{6}$ Lopransi S, Mihm MC. Pilomatrix carcinoma or calcifying epitheliocarinoma of Malherbe. A case report and review of the literature. Cancer 1980; 45: 2368-2373

7 De Galvez-Aranda MV, Herrera-Ceballos E, Sanchez-Sanchez P, BoschGarcia RJ, Matilla-Vicente A. Pilomatrix carcinoma with lymph node and pulmonary metastasis: report of a case arising on the knee. Am J Dermatopathol 2002; 24 (2): 139-43

${ }^{8}$ Weedon D, Bell J, Mayze J. Matrical carcinoma of the skin. J Cutan Pathol 1980; 7: 39-42

${ }^{9}$ Bridger L, Koh HK, Smiddy M et al. Giant pilomatrix carcinoma: report and review of the literature. J Am Acad Dermatol 1990; 23: 985 - 988

${ }^{10}$ Marrogi AJ, Wick MR, Dehner LP. Pilomatrical neoplasms in children and young adults. Am J Dermopathol 1992; 2: 87-94

${ }^{11}$ Haferkamp B, Bastian BC, Bröcker EB et al. Pilomatrixkarzinom in ungewöhnlicher Lokalisation. Fallbericht und Literaturübersicht. Hautarzt 1999; 50: 355-359

12 Tateyama H, Eimoto T, Tada T et al. Malignant pilomatrixoma. An immunohistochemical study with antihair keratin antibody. Cancer 1992; 69: 127 - 132

${ }^{13}$ Van der Walt JD, Rohlova B. Carcinomatous transformation in a pilomatrixoma. Am J Dermatopathol 1984; 6: 63-69

${ }^{14}$ Gould E, Kurzon R, Kowalczyk AP et al. Pilomatrix carcinoma with pulmonary metastasis. Report of a case. Cancer 1984; 54: 370 - 372

${ }^{15}$ Wood MG, Parhizgar B, Beerman H. Malignant pilomatrixoma. Arch Dermatol 1984; 120: 770-773

${ }^{16}$ Veliath A, Reddy K, Gomathinayagam D. Malignant pilomatrixoma: report of a case. Acta Radiol Oncol 1984; 23: 429-431

17 Manivel C, Wick MR, Mukai K. Pilomatrix carcinoma: an immunohistochemical comparison with benign pilomatrixoma and other benign cutaneous lesions of pilar origin. J Cutan Pathol 1986; 13: 22 - 29 
18 Mir R, Cortes E, Papantoniou PA et al. Metastatic trichomatrical carcinoma. Arch Pathol Lab Med 1986; 110: 660-663

${ }^{19}$ Chen K, Taylor D. Pilomatrix carcinoma. J Surg Oncol 1986; 33: 112 114

${ }^{20}$ Green E, Sansusi D, Fowler M. Pilomatrix carcinoma. J Am Acad Dermatol 1987; 17: $264-270$

${ }^{21}$ Miyahara H, Imayama S, Hasizume T et al. Two cases of pilomatrix carcinoma. J Dermatol 1990; 17: 322 - 325

22 Masahashi T, Takahashi S, Maie O. Pilomatrix carcinoma report of the second case in Japan and a review of the literature. Nippon Hifuka Gakkai Zasshi 1990; 100: 533-534

${ }^{23}$ Zagarella SS, Kneale KL, Stern HS. Pilomatrix carcinoma of the scalp. Australas J Dermatol 1992; 33: 39-42

24 Sloan JB, Sueki H, Jaworsky C. Pigmented malignant pilomatrixoma: report of a case and review of the literature. J Cutan Pathol 1992; 19: $240-246$

25 Sau P, Lupton GP, Graham JH. Pilomatrix carcinoma. Cancer 1993; 71 : $2491-2498$

26 O’Donovan DG, Freemont AJ, Adams JE et al. Malignant pilomatrixoma with bone metastasis. Histopathology 1993; 23: 385- 386

${ }^{27}$ Hanly MG, Allsbrook WC, Pantazis CG et al. Pilomatrical carcinosarcoma of the cheek with subsequent pulmonary metastases. A case report. Am J Dermatopathol 1994; 16: 196-200

28 Sabhaward BD, Malhotra V. Pilomatrix carcinoma calcifying epitheliocarcinoma of Malherbe (a case report). Indian J Pathol Microbiol 1994; 37 (suppl): $45-46$

${ }^{29}$ Monchy D, McCarthy SW, Dubordieu D. Malignant pilomatrixoma of the scalp. Pathology 1995; 27: $201-203$

${ }^{30}$ Niedermeyer HP, Peris K, Hofler H. Pilomatrix carcinoma with multiple visceral metastases. Report of a case. Cancer 1996; 77: 1311 -1314

31 Vico P, Rahaier Y, Granem G et al. Pilomatrix carcinoma. Eur J Oncol 1997; 23: 370- 371

$32 \mathrm{Li} \mathrm{X}$, Jiang H, Li A. Clinicopathological study on 15 cases of pilomatrix carcinoma. Chung Hua Ping Li Hsueh Tsa Chih 1997; 26: 100-102

33 Waxtein L, Vega E, Alvarez L et al. Malignant pilomatrixoma: a case report. Int J Dermatol 1998; 27: 538-540

34 Deolekar MV, Brown DC, Desai SA. Malignant pilomatrixoma: a case report with fine needle aspiration (FNA) cytology. Cytopathology 1999; 10: 270-275

${ }^{35}$ Caubet J, Ramos R, Ortabe I et al. Pilomatrix carcinoma of the face. J Oral Maxillofac Surg 1999; 57: 609-611

${ }^{36}$ Cahill MT, Moriarty PM, Mooney DJ et al. Pilomatrix carcinoma of the eyelid. Am J Ophthalmol 1999; 127: $463-464$

37 Panico L, Manivel JC, Pettinato G et al. Pilomatrix carcinoma. A case report with immunohistochemical findings, flow cytometric comparison with benign pilomatrixoma and review of the literature. Tumori 1994; 80: 309-314

${ }^{38}$ Sassmannshausen J, Chaffins M. A report of a case arising from previously excised pilomatrixoma and review of the literature. J Am Acad Dermatol 2001; 44: 358-361

${ }^{39}$ Hardisson D, Linares MD, Cuevas-Santos J, Contreras F. Pilomatrix carninoma: a clinicopathologic study of six cases and review of the literature. Am J Dermatopathol 2001; 23 (5): 394-401

${ }^{40}$ Petit T, Grossin M, Lefort E, Lamarche F, Hénin D. Pilomatrcome malin: aspects histologiques et immunhistochimique. Ann Pathol 2003; 23: $50-54$

${ }^{41}$ Mack U, Back W, Reiter S. Pilomatrixkarzinom mit polytoper Metastasierung nach 23 Jahren. Akt Dermatol 1994; 20: 351 - 355

\section{Buchbesprechung}

\section{Venenerkrankungen. Wirksame Hilfe bei Besenreisern, Krampfadern, Thrombose und offenem Bein}

M. Stücker, S. Reich, P. Altmeyer

Stuttgart: Kohlhammer, 2004. 264 S. Kart. 22,- $€$.

ISBN 3-17-017586-6

In der Kohlhammer Taschenbuchreihe „Rat \& Hilfe“ ist als 21. Band derjenige über Venenerkrankungen aus der Feder des Venen-Teams der Universitäts-Klinik für Dermatologie und Allergologie der Ruhr-Universität Bochum erschienen. Um es vorwegzunehmen: ein prima Taschenbuch, hervorragend geeignet und ausgerichtet auf die Patienten und deren Angehörige. Es ist eine Freude, darin zu lesen! Dieses Taschenbuch kann man uneingeschränkt seinen Patienten empfehlen. Aus der Venen- und Beinsprechstunde hervorgegangen, zeugt es vom regen Zulauf derselben und von einfühlsamen Ärzten. Auf 261 Seiten wird den Patienten Antwort auf alle Fragen geboten. Und dies in präziser, einfach gehaltener und sachdienlicher Sprache. Keine Einseitigkeit, keine Doktrin und keine Mission, aber breit angelegte und klare Information. Eine echte Hilfe, ein wahrer Leitfaden und ein guter Ratgeber.

Alle wichtigen und immer wieder gestellten Fragen sind aufgegriffen und sie werden in Wort und Bild, gelegentlich durch eine Tabelle, oft durch anschauliche Zeichnungen ergänzt, hervorragend beantworten. Kurz aber präzise!

Den Krankheitsbegriffen sind Ausführungen über Aufbau, Funktion und Diagnostik vorangestellt, immer im bewährten FrageAntwort Wechselspiel und sinnhaft aufgereiht. Die Therapie als Herzstück spricht alles an, was in Frage kommt und die Patienten interessiert. Eine Randbemerkung: die biochirurgische Ulkusreinigung mit Fliegenmaden wird zwar auf Seite 159 erwähnt (nicht im Sachregister), dann aber nicht weiter ausgeführt. So spielen doch regionale Aspekte immer mit, wären doch diesbezügliche Fragen am Oberrhein oft und facettenreich angefallen.

Es folgen Abschnitte über Prophylaxe, Venenleiden in Schwangerschaft und Wochenbett und ein solcher zum wichtigen Thema „Leben mit einer Venenerkrankung“. Stichwortverzeichnis, Erklärung medizinischer Fachbegriffe, Literatur, wichtig Anschriften und einige gesetzliche Grundlagen ergänzen und bereichern das Taschenbuch in bester Weise.

Dank an die Autoren, Empfehlung an die Patienten und der Wunsch nach weiter Verbreitung des Buches kommen von Herzen.

E. G. Jung, Heidelberg 